\title{
FreeStyle Libre: available on the NHS?
}

\author{
THOMAS SJ CRABTREE, ${ }^{1}$ THOZHUKAT SATHYAPALAN, ${ }^{2}$ EMMA G WILMOT ${ }^{1}$
}

\begin{abstract}
In 2017 the FreeStyle Libre flash glucose monitor became available on the NHS Drug Tariff. Clinicians and funders across the UK have had to debate where this device sits in the care of people with diabetes. While some would argue that this device is a replacement for self-monitoring of blood glucose and should therefore be widely available for those who may benefit, the financial climate in the NHS has demanded a more conservative approach. This article considers the current evidence to support the use of the FreeStyle Libre, its position in current and future care pathways and gaps in available evidence.
\end{abstract}

Br J Diabetes 2018;18:3-6

Key words: flash glucose monitoring, FreeStyle Libre, type 1 diabetes, interstitial glucose monitoring, funding, reimbursement

\section{Introduction}

Self-monitoring of blood glucose (SMBG) is an integral component of the self-management of type 1 diabetes. In 2015 the FreeStyle Libre (FSL) device was introduced as a potential replacement for SMBG. It is a round white disc worn on the arm which continuously senses interstitial glucose (Figure 1). Users scan the disc with their phone or a reader to discover the trajectory of their glucose levels, taking into account the 5-minute lag between blood and interstitial glucose. However, it is not continuous glucose monitoring (CGM); it does not alarm to alert the user to rising or falling glucose levels. Overall, the ease of use, low cost compared to CGM, availability of free trial samples from the company and the option for direct consumer online purchase has led to a heightened demand for this novel technology in the UK. In November 2017 FSL became available on the NHS drug tariff, although whether this will pave the way to access for those living with diabetes remains to be seen. This paper briefly summarises the benefits, available evidence and considers the role of the FSL in current clinical pathways. A more detailed review of the

Derby Teaching Hospitals NHS Foundation Trust, Derby, UK Academic Diabetes, Endocrinology and Metabolism, Hull York Medical School, University of Hull, Hull, UK

Address for correspondence: Dr Emma Wilmot Derby Teaching Hospitals NHS Foundation Trust, Derby, DE22 3NE, UK E-mail: emma.g.wilmot@gmail.com

https://doi.org/10.15277/bjd.2018.168
Figure 1. FreeStyle Libre reader and sensor (www.freestylelibre.co.uk)

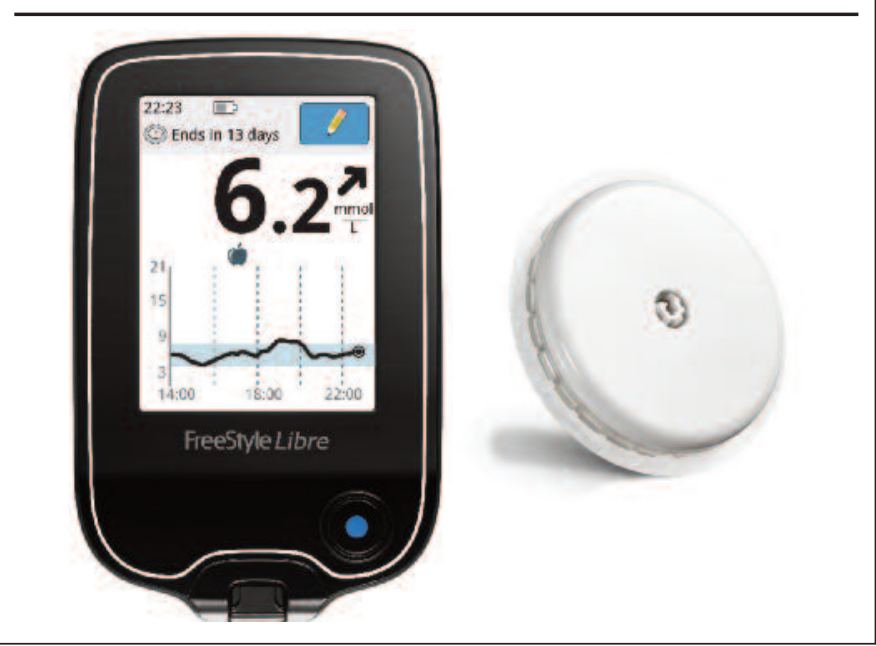

evidence for the device is available in the paper by Leelarathna and Wilmot. ${ }^{1}$

\section{User satisfaction}

It seems intuitive that people with diabetes might prefer the FSL to continuously pricking their fingers to obtain samples for blood glucose readings, in addition to the ability to view the direction and rate of change of glucose levels. Indeed, several studies have confirmed high levels of patient satisfaction with the FSL in adult and paediatric populations..$^{2-4}$ The IMPACT study, the major randomised controlled trial assessing the FSL, also described higher rates of treatment satisfaction. ${ }^{5}$

Randomised controlled trial and observational study data In randomised controlled trials in both type 1 and type 2 diabetes, the FSL has demonstrated improvements in glucose end points including less time in hypoglycaemia and improved time in range, although to date no randomised controlled trial has demonstrated a reduction in $\mathrm{HbA}_{1 \mathrm{c}}$.

The IMPACT randomised controlled trial ${ }^{5}$ included people with tightly controlled type 1 diabetes who were using either multiple daily injections or continuous subcutaneous insulin infusions. FSL use led to a -1.39 hour reduction in the time spent in hypoglycaemia compared with -0.14 hours in the control group $(p<0.0001)$. Given this $38 \%$ reduction in hypoglycaemia, an increase in $\mathrm{HbA}_{1 c}$ may have been anticipated. However, the $\mathrm{HbA}_{1 c}$ remained static in the FSL group, a reflection of reduced hypergly- 
Table 1 Summarising the outcomes of observational studies assessing the FSL; absolute HbA 1 c reductions DCCT(\%)

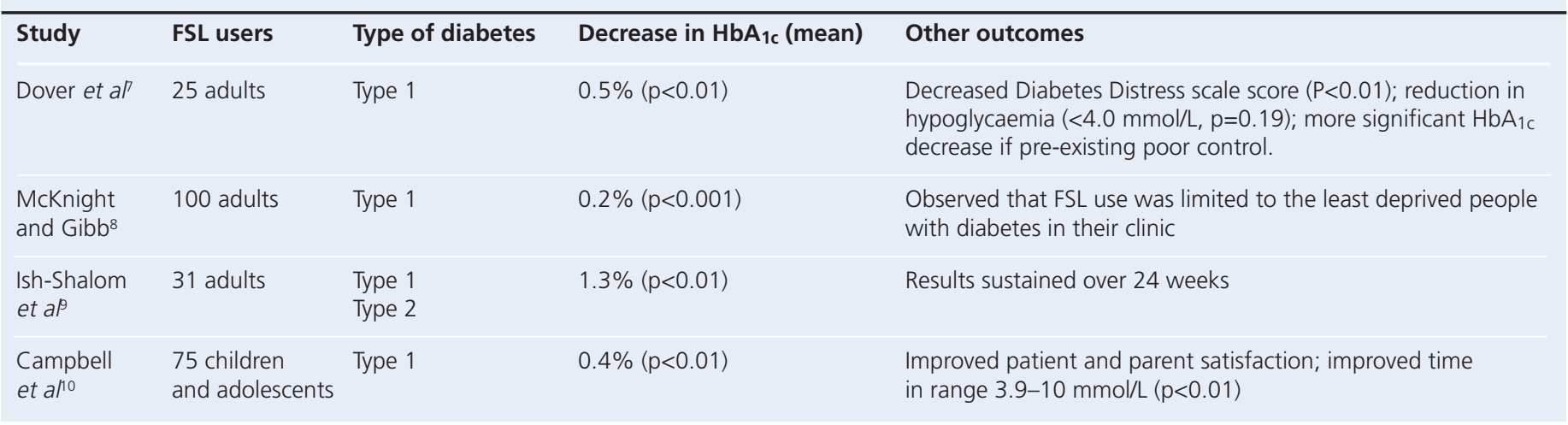

caemia and increased time in range. These changes were achieved within a fairly short time period with minimal use of finger-prick blood testing (on average one test every 2-5 days), providing reassuring data for the use of the FSL for insulin dosing decisions, otherwise known as non-adjunctive use. In the USA and Europe the FSL and the Dexcom G5 are now approved for non-adjunctive use.

There are also data to support the use of FSL as a replacement for SMBG in people with type 2 diabetes undergoing intensive insulin therapy. A study by Haak et a/ ${ }^{6}$ showed significant reductions in the time spent in hypoglycaemia by an average of $48 \%$, with just eight scans a day. Although again no reduction in $\mathrm{HbA} 1 \mathrm{c}$ was seen, the FSL is a safe alternative to capillary blood glucose measurements in this cohort.

Further observational studies over longer time frames have demonstrated reductions in $\mathrm{HbA}_{1 \mathrm{c}}$ and, as might be expected, these reductions were greater in those who had poor glycaemic management to start with. The findings of these studies are summarised in Table 1.

\section{Clinical use of the FreeStyle Libre}

The data produced by the FSL provide insight into glucose levels over 24 hours of each day and can be downloaded via Abbott software or Diasend for review in the clinic. The user can also display FSL data on either the reader or their smartphone to reveal not only glucose data but also trends and arrows indicating the rate of change in glucose. The intuitive nature of this data is supported by the reductions in hypoglycaemia in the IMPACT trial, ${ }^{5}$ which were witnessed within two weeks of use without any formal training. It should be noted that the FSL device cannot currently be used for glucose monitoring in the context of driving, although interestingly the DVLA have recently removed the term 'blood' from their guidance on glucose monitoring, potentially paving the way for other methods of glucose monitoring in the future. Blood glucose is also the preferred method of glucose monitoring in the following circumstances: (1) when glucose levels are rapidly changing; (2) if hypoglycaemia or impending hypoglycaemia is displayed; (3) when scanned glucose results do not correspond with user symptoms.

\section{Adverse events}

Adverse events are almost universally related to the adhesive used on the sensor and were uncommon. Severe reactions (defined as lasting $>7$ days or requiring medical intervention) noted during the IMPACT trial ${ }^{5}$ were found to be easily manageable either by relocation of the sensor or the use of barrier or medicated creams.

\section{FreeStyle Libre versus continuous glucose monitoring (CGM) devices}

Some may question the use of the FSL over conventional CGM, and there are situations in which CGM is notably superior. People with diabetes with impaired awareness of hypoglycaemia or who experience intractable, severe and unpredictable hypoglycaemic episodes are more likely to be reliant on CGM sensor alarms to alert them to impending low glucose levels, and this is a feature which is not present on the FSL. In this situation, it would seem sensible to opt for CGM rather than the FSL, in line with the guidance of the National Institute for Health and Care Excellence (NICE). ${ }^{11}$ Indeed, evidence from Professor Oliver's group in London comparing the FSL with real-time CGM (Dexcom G5) in patients with impaired hypoglycaemic awareness showed that, while both helped improve time spent within range, the Dexcom $\mathrm{G} 5$ was superior in preventing hypoglycaemia at all levels as well as their pre-defined primary end point of preventing glucose levels of $<3.3 \mathrm{mmol} / \mathrm{L}$. Notably, CGM also improved fear of hypoglycaemia, as evidenced by decreased hypoglycaemia fear from baseline. ${ }^{12}$ It should be noted that baseline data for both groups were collected using Dexcom G5 but follow-up data were collected on the FSL in the FSL group and Dexcom G5 in the Dexcom group, which limits interpretation of the findings.

One possible advantage of the FSL over CGM is that there is no requirement for calibration of the FSL. The FSL is factory calibrated and therefore does not require a daily or twice daily calibration against a simultaneous finger-prick blood glucose reading. This makes it much simpler to use and removes the errors that may be introduced by user variability in finger-prick testing technique, such as not washing hands prior to finger stick testing or calibrating when glucose is in a state of flux and the difference between sensor and blood glucose is at its greatest. ${ }^{13}$

Overall, the reported accuracy of the FSL is similar to other CGM systems. One open-label trial has compared the accuracy of the FSL and Dexcom and Medtronic Enlite sensors. The FSL was more accurate than the Medtronic and Dexcom sensors, with significantly lower mean average relative difference both in extremes of 
glycaemia and during exercise, using venous blood glucose sampling as the standard. ${ }^{14}$ Further head to head studies are required.

\section{Positioning FSL in the clinical pathway}

Based on the IMPACT trial data, FSL is a safe alternative to SMBG. The Diabetes UK position statement recommends its use for all people with diabetes who are treated with intensive insulin therapy. ${ }^{15}$ If cost was not a consideration, wider access would seem appropriate. Although the FSL is considerably less expen-

Box 1 Excerpt from Regional Medicine Optimisation Committee statement regarding FreeStyle Libre ${ }^{17}$

It is recommended that FreeStyle Libre should only be used for people with type 1 diabetes, aged four and above, attending specialist Type 1 care using multiple daily injections or insulin pump therapy, who have been assessed by the specialist clinician and deemed to meet one or more of the following:

1. Patients who undertake intensive monitoring $>8$ times daily

2. Those who meet the current NICE criteria for insulin pump therapy $\left(\mathrm{HbA}_{1 \mathrm{c}}>8.5 \%(69.4 \mathrm{mmol} / \mathrm{mol})\right.$ or disabling hypoglycaemia where a successful trial of FreeStyle Libre may avoid the need for pump therapy.

3. Those who have recently developed impaired awareness of hypoglycaemia. It is noted that for persistent hypoglycaemia unawareness, NICE recommend continuous glucose monitoring with alarms and FreeStyle Libre does not currently have that function.

4. Frequent admissions ( $>2$ per year) with DKA or hypoglycaemia

5. Those who require third parties to carry out monitoring and where conventional blood testing is not possible.

In addition, all patients (or carers) must be willing to undertake training in the use of FreeStyle Libre and commit to ongoing regular follow-up and monitoring (including remote follow-up where this is offered). Adjunct blood testing strips should be prescribed according to locally agreed best value guidelines with an expectation that demand/frequency of supply will be reduced. sive than CGM, it is not yet cost comparable with SMBG unless $\geq 8$ capillary blood glucose checks are performed per day. Although NICE recommends 4-10 blood glucose checks per day, in reality few people with diabetes achieve this. Given the potential excess costs and the current NHS financial climate, cost effectiveness is a priority. The NICE MIB ${ }^{16}$ identified gaps in the current evidence base relating to the FSL and the long-term implications of use, both in terms of clinical outcomes and costs. With the FSL available on the drug tariff but no clear guidance on which groups might benefit most, there was concern that variation in access to this novel technology may contribute to further geographical variation in outcomes.

In an attempt to prevent the development of a postcode lottery for FSL access, the Regional Medicine Optimisation Committee (RMOC) published national recommendations for reimbursement (see Box 1). ${ }^{17} \mathrm{~A}$ key requirement of the RMOC recommendations was the ability to audit outcomes in users of the FSL.

As such, ABCD have established a national FSL audit tool (Figure 2), allowing centres to submit anonymised data from their users to be analysed both centrally and locally to provide funders with realworld data on clinical outcomes such as $\mathrm{HbA}_{1 c}$ change, hypoglycaemic awareness and frequency, diabetes distress. If real-world benefits can be demonstrated, this may pave the way for wider reimbursement in the future.

Although randomised controlled trial data to demonstrate change in $\mathrm{HbA}_{1 c}$ are lacking and current criteria are restrictive, we have reached a pivotal point in the management of type 1 diabetes. It is difficult to imagine that a decade from now we will still be recommending 4-10 painful blood glucose tests per day for people with type 1 diabetes when such affordable, insightful and more humane alternatives exist.

\section{Conclusion}

The FSL represents possibly the greatest and most innovative change in diabetes care since home blood glucose monitoring was

Figure 2. ABCD FreeStyle Libre Audit, available at: http://www.diabetologists-abcd.org.uk/n3/FreeStyle_Libre_Audit.htm

\section{Association of British Clinical Diabetologists}

\section{FreeStyle Libre Nationwide Audit}

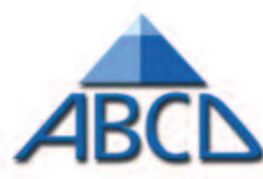

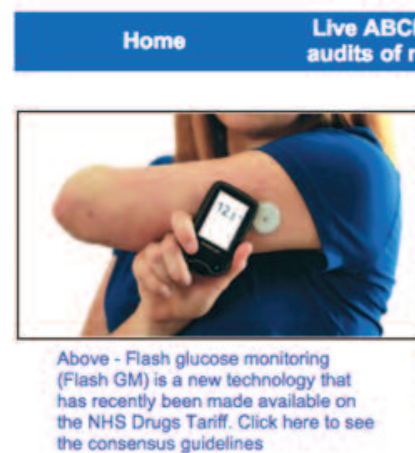

the consensus guidelines
All ABCD nationwide audits

Future audits of new theraples

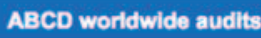

ABCD nationwide FreeStyle Libre (FSL) audit on N3

\section{About the $A B C D$ nationwide FSL audit}

With the launch of the FSL audit, $A B C D$ is now auditing the use of FSL in adults and paediatric patents with both type 1 and type 2 diabetes. On the left you will find examples of findings from randomised controlled trials (RCTs) undertaken with FSL. As with the other $A B C D$ audits ( for example exenatide, liraglutide, dapagliflozin), it may be that outcomes for patients using FSL in real clinical practice will be different from those in the clinical trials. It is the aim of this audit to find this out. As with other

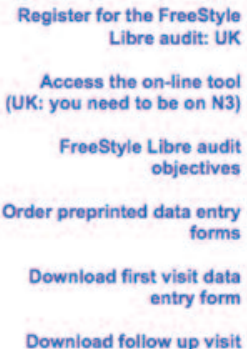




\section{Key messages}

- The FreeStyle Libre is an arm worn, calibration free, interstitial glucose monitor

- The IMPACT randomised controlled trial demonstrated a reduction in hypoglycaemia and high treatment satisfaction in people living with Type 1 diabetes

- The FreeStyle Libre became available on the NHS Drug Tariff in November 2017. The Regional Medicines Optimisation Committee (RMOC) have made recommendations for reimbursement in England

- $A B C D$ plan to independently collate real world data on FreeStyle Libre use through their nationwide data. UK clinicians from both adult and paediatric services can input to the audit

first introduced. While there may be gaps in the available evidence and long-term benefits, what is clear is that this is a desirable device for many people living with diabetes, as evidenced by reports on user satisfaction and the sheer volume of those willing to self-fund the device. While national recommendations have been published to guide reimbursement, at the time of writing it is not yet clear whether these will translate into access to the device for these specified groups of people with diabetes.

\section{Web links for further information}

For further information on the FreeStyle Libre and to gain an insight into the interface used, ${ }^{18}$ visit https://www.freestylelibre.co.uk/libre/

Conflict of interest EW has received personal fees from Dexcom, Medtronic and Abbott Diabetes Care.

\section{Funding None received.}

\section{References}

1. Leelarathna L, Wilmot EG. Flash forward: a review of flash glucose monitoring. Diabet Med 2018 Jan 22. http://dx.doi.org/10.1111/dme.13584

2. Olafsdottir AF, Attvall $S$, Sandgren $U$, et al. A clinical trial of the accuracy and treatment experience of the flash glucose monitor FreeStyle Libre in adults with type 1 diabetes. Diabetes Technol Ther 2017;19:164-72. http://dx.doi.org/10.1089/dia.2016.0392.

3. Edge J, Acerini C, Campbell F, et al. An alternative sensor-based method for glucose monitoring in children and young people with diabetes. Arch Dis Child 2017;102:543-9. http://dx.doi.org/10.1136/archdischild-2016-311530

4. McPhater A, Gardiner M, Modgil G. The impact of the Libre device for families and children with type 1 diabetes (abstract). Diabet Med 2017;34(Suppl S1):104.

5. Bolinder J, Antuna R, Geelhoed-Duijvestijn P, Kroger J, Weitgasser R. Novel glucose-sensing technology and hypoglycaemia in type 1 diabetes: a multicentre, non-masked, randomised controlled trial. Lancet 2016;388:225463. http://dx.doi.org/10.1016/s0140-6736(16)31535-5

6. Haak T, Hanaire H, Ajjan R, Hermanns N, Riveline JP, Rayman G. Use of flash glucose-sensing technology for 12 months as a replacement for blood glucose monitoring in insulin-treated type 2 diabetes. Diabetes Ther 2017;8:573-86. http://dx.doi.org/10.1007/s13300-017-0255-6.

7. Dover AR, Stimson RH, Zammitt NN, Gibb FW. Flash glucose monitoring improves outcomes in a type 1 diabetes clinic. J Diabetes Sci Technol 2017;11:442-3. http://dx.doi.org/10.1177/1932296816661560

8. McKnight JA, Gibb FW. Flash glucose monitoring is associated with improved glycaemic control but use is largely limited to more affluent people

in a UK diabetes centre. Diabet Med 2017;34:732.

http://dx.doi.org/10.1111/dme.13315

9. Ish-Shalom M, Wainstein J, Raz I, Mosenzon O. Improvement in glucose control in difficult-to-control patients with diabetes using a novel flash glucose monitoring device. J Diabetes Sci Technol 2016;10:1412-13. http://dx.doi.org/0.1177/1932296816653412

10. Campbell F, Kordonouri O, Murphy N, Stewart C. FreeStyle Libre use for selfmanagement of diabetes in children and adolescents. Diabetes 2017 66(Suppl 1A):LB28.

11. National Institute for Health and Care Excellence. Type 1 diabetes in adults: diagnosis and management. https://wwwniceorguk/guidance/ng17 2015.

12. Reddy M, Jugnee N, Spanudakis E, Oliver N. Impact on hypoglycaemia awareness of real time continuous glucose monitoring and intermittent continuous glucose data (I HART CGM). Diabetic Med 2017;34:29.

13. Hoss $U$, Budiman ES. Factory-calibrated continuous glucose sensors: the science behind the technology. Diabetes Technol Ther 2017;19:S44-S50. http://dx.doi.org/10.1089/dia.2017.0025

14. Aberer F, Hajnsek M, Rumpler M, et al. Evaluation of subcutaneous glucose monitoring systems under routine environmental conditions in patients with type 1 diabetes. Diabetes Obes Metab 2017;19:1051-5. http://dx.doi.org/10.1111/dom.12907

15. Diabetes UK. Consensus guideline for flash glucose monitoring systems. https://www. diabetes.org.uk/resources-s3/2017-09/1190_Flash\%20glucose\%20monitoring\%20guideline_SB_V9\%5B4\%5D.pdf 2017

16. National Institute for Health and Care Excellence. FreeStyle Libre for glucose monitoring. https://wwwniceorguk/advice/mib110 2017

17. Regional Medicines Optimisation Committee NHS England. Flash glucose monitoring systems - position statement. https://www.sps.nhs.uk/wpcontent/uploads/2017/11/Flash-Glucose-monitoring-System-RMOCStatement-final-2.pdf 2017

18. Abbott Laboratories. FreeStyle Libre. www.freestylelibre.co.uk/libre (accessed 22 Feb 2018).

\section{Did you know incidence of} type 1 diabetes is increasing by $4 \%$ each year?

We know that type 1 diabetes is an auto immune condition and is not caused by anything that the person did or did not do. JDRF, the type 1 diabetes charity, exists to cure, treat and prevent type 1 diabetes and we offer FREE resources and events to those living with the condition.

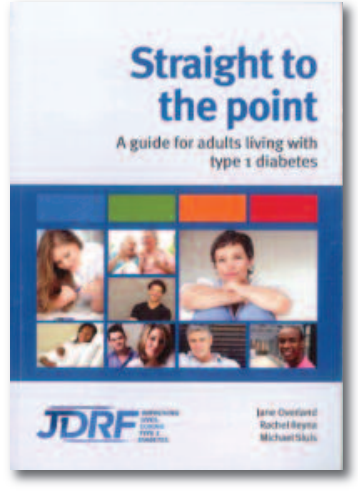

To order FREE information and resources for your patients with type 1 diabetes go to jdrf.org.uk/hp

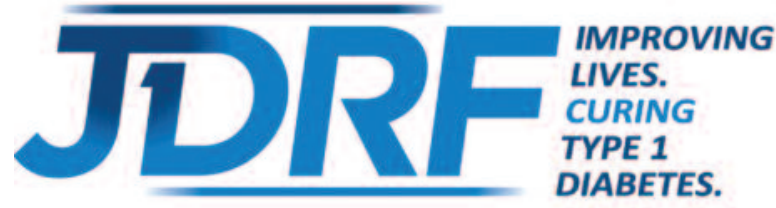

